\title{
Non-Occluding Intelligent Magnifiers for Sketching on Small Displays
}

\author{
Paul Schmieder, Beryl Plimmer \\ Department of Computer Science \\ University of Auckland \\ Private Bag 92019 \\ Auckland, New Zealand \\ psch068@aucklanduni.ac.nz, beryl@cs.auckland.ac.nz, john.hosking@anu.edu.au \\ John Hosking \\ ANU College of Engineering and Computer \\ Science \\ Australian National University \\ Canberra, ACT 0200 \\ Australia
}

We present intelligent magnification techniques that combine distortion techniques with automatic content information awareness. They have been developed to support sketching on small displays. A first evaluation study that tested and compared three existing distortion lenses showed their general unsuitability for sketching. Based on these findings we developed and evaluated a new set of intelligent lenses, which use context information as the distortion regulator. The evaluation shows that using our new intelligent lenses leads to fewer errors, without time penalties, and with higher user satisfaction.

Digital sketching, sketch tools, focus and context techniques, sketch recognition algorithm.

\section{INTRODUCTION}

In this paper we investigate how distortion based magnification lenses can be used to support sketching on stylus input devices with small displays. Digital sketching is potentially more powerful than pen and paper sketching as it provides the means to manipulate content. Zooming, rotating, scaling, relocating and erasing are only a fraction of the possible basic editing features a digital environment has to offer. More advanced editing techniques include beautification (Plimmer, Purchase et al. 2010), recognition and various transformations to other representations (Plimmer and Freeman 2007). However, limited display size (Gatward 2008) reduces interaction capabilities.

To date, little research has been done into ways to support a canvas that is larger than the available display space on small displays. Lack of space could be because of a very large canvas, or because of a requirement for a high detail sketch on a small canvas. What is required is a way to sketch at a higher resolution than the base canvas. A common approach is to magnify the canvas. Consider the sketch in Figure 1 (a); two common strategies are to zoom the canvas or use a magnification lens. A zoomed canvas strategy only displays a portion of the sketch as shown in Figure 1 (b). Mechanisms for navigation such as panning, scrolling and paging must be used to select which part of the magnified information to display. A magnifying lens strategy leaves the remaining canvas unchanged but still occludes information;

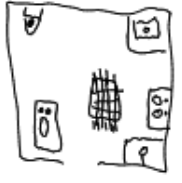

(a)

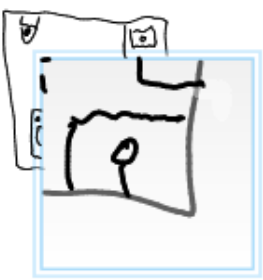

(c)

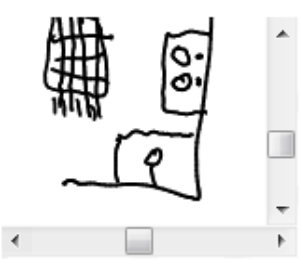

(b)

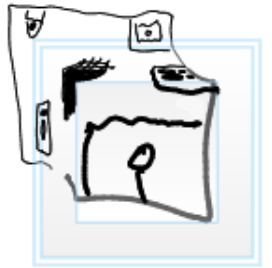

(d)

Figure 1: The same sketch are shown with (a) no magnification, (b) being zoomed in and scrollbars for navigation, (c) a magnifying lens occluding its immediate surroundings and (d) a fisheye lens which distorts the content surrounding the focus area.

i.e. the information directly surrounding the magnified region (Figure 1 (c)).

To fully comprehend a sketch, all its information pieces have to be understood and their place within the information structure has to be known. Both magnification strategies (Figure $1(b+c)$ ) occlude content, and manual navigation is necessary to view occluded information. The extra steps needed for navigation can be frustrating and also increase mental workload (Grudin 2001). In addition, if only a small part of the sketch is visible, the user has to mentally visualize the information structure based on his/her working memory (Wickens and Hollands 
2000) which also increases mental workload. To fully comprehend a sketch the complete content has to be shown at all times.

Distortion lenses, such as fisheye lenses (Furnas 1986), overcome the problem of occlusion (Figure 1 (d)). To generate the space required by the magnified content, parts of the information are visually transformed using dimensions such as space (Furnas 1986), speed of movement (Gutwin 2002) and translucency (Pietriga and Appert 2008). A comparison of fisheye lenses and scrolling interfaces on small displays showed participants preferred a distortion lens (Lank and Phan 2004).

We describe two studies evaluating both existing and new lenses. In the first, we test standard distortion lenses; i.e. fisheye (Furnas 1986) and blending lenses (Pietriga and Appert 2008). We found these confuse participants as the magnified region is moving and the ink in the distorted region is difficult to understand. This left the participants unwilling to continue with the lenses. We devised new intelligent, fisheye like lenses to overcome the problems identified and repeated the evaluation with our intelligent lenses, with more success.

Our contributions are a better understanding of the challenges of using lenses for creating and editing content, and techniques behind our novel lenses which can be applied to other problems involving content creation on small displays.

\section{RELATED WORK}

There are a variety of strategies to support magnification on digital displays. Overview + detail and zoom strategies occlude content while showing elements magnified, while focus + context strategies show the complete content. The latter techniques, often referred to as distortion techniques, generate the space required to show parts of the information magnified by visually transforming the information.

There are various distortion techniques and domains they have been applied to. Techniques range from fisheye lenses (Furnas 1986), the document lens (Robertson and Mackinlay 1993), speed coupled lenses (Gutwin 2002), sigma lenses (Pietriga and Appert 2008) and blurring lenses (Kosara, Miksch et al. 2001). The performances of these techniques differ depending on the domain which vary from navigation (Baudisch, Good et al. 2002) to reading comprehension (Hornbaek and Frokjaer 2003) and graph editing (Schaffer, Zuo et al. 1996). While distortion lenses increase performance on tasks such as target acquisition (Ramos, Cockburn et al. 2007) and large steering

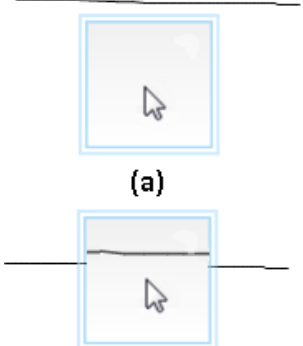

(c)

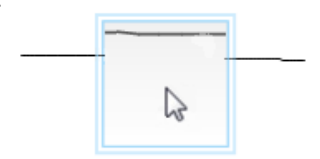

(b)

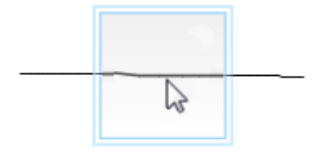

(d)
Figure 2: The moving canvas problem. The cursor is always the lens center. As the cursor moves towards the line $(a-c)$, the magnified part of the line moves towards the cursor. Both meet at the line's original position (d).

(Gutwin and Skopik 2003), they negatively impact performance of menu selection (Bederson 2000).

Magnification lenses using distortion techniques have several problems. One is additional mental workload required by the user to understand and interact with the distorted ink; e.g. understanding spatial relationships between distorted objects can be difficult (Carpendale, Cowperthwaite et al. 1997). The moving canvas (Figure 2) is another problem caused by some distortion lenses such as the fisheye. It is caused by scale differences between visual and motor space in the magnified region (Gutwin 2002). Appert et al. (2010) decoupled lens and cursor allowing for pixel precise pointing.

The practical advantages and effectiveness of applying zooming techniques to sketching have been shown by Agrawala and Shilman (2005). In their interface a region of the document is magnified to allow annotations to be written at the same size as the document's content.

While Agrawala and Shilman (2005) have used a zoom region which occludes parts of the content, Lank and Phan (2004) have employed a fisheye lens to avoid hiding context. Due to limited hardware performance their lens remains where it is activated rather than following the stylus. Using a static lens has helped them to avoid the problems faced by movable lenses such as moving objects. Their evaluation study compared a traditional zooming interface and a fisheye lens. The lens was, overall, perceived positively and despite the static behaviour participants preferred the fisheye lens over having to scroll the canvas for their drawing tasks (Lank and Phan 2004).

In sum, the tasks distortion lenses have been tested on almost exclusively concern viewing and selecting content. Their potential to allow users to rapidly create and edit content with high precision has been previously implied; i.e. evaluations have shown the magnification lenses' suitability for 
sketching and successfully compared them with traditional zooming interfaces. However, due to the versatile nature of sketching, both the known difficulties and possible emerging ones mean that applying distortion lenses to sketching poses challenges which have to be overcome. To understand these challenges better we undertook a user study evaluating existing lens techniques.

\section{TESTING EXISTING LENSES FOR SKETCHING}

We conducted an experiment asking participants to complete four tasks using three different distortion lenses: fisheye (Furnas 1986), blending (Pietriga and Appert 2008) and fixed fisheye lens (Furnas 1986). We implemented these lenses into our test environment. This is written in $\mathrm{C \#}$ using the Microsoft Windows Real-Time Stylus interface, with rendering partly done on the GPU using Direct2D for real time performance.

To capture input a digital stylus is used. The stylus can be detected on the surface and immediately above it. If the stylus is hovering over the surface, the distortion lens is shown relative to the stylus tip's position. This is used to navigate the canvas and inspect the sketched objects. When in contact with the surface digital ink is generated. Lens and stylus are coupled thus when the stylus is moved, the lens moves as well.

The goal of this evaluation was to investigate how existing distortion lenses perform in the sketching domain. Magnification lenses in combination with distortion techniques have never been applied to sketching. As we will show they are not usable for sketching. However, this study provides insights into the problem and provides baseline performance data.

\subsection{Setup}

Our study had a within-subject design and tested two variable factors: distortion lens (fisheye, blending, fixed lens) and task type (drawing, writing). Participants completed three task series, each comprising two drawing and two writing tasks. The order of task series was identical for each participant: each started with series one and finished with series three. To reduce the learning effect, the tasks had a similar design across the three series; e.g. the first task in each series was to complete a different floor plan. The order of lenses was varied forming six conditions. This allowed us to account for the possible impact of lens order.

Twelve participants volunteered for our study (10 male and 2 female), ranging in age from 20 to 32 years. None had used digital distortion lenses before. Two of the participants were left handed.

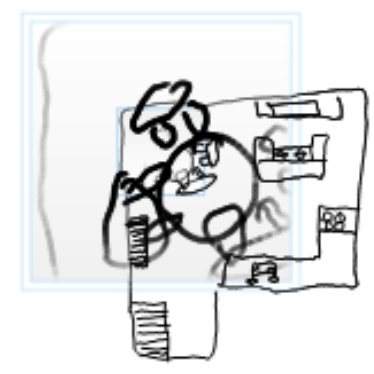

Figure 3: Blending lens: The upper layer shows the ink fully opaque in the center and linearly fades out in the outer lens. The lower layer shows the unmagnified content which fades out towards the lens center.

Participants used a HP EliteBook 2740p tablet PC with a 13" display of resolution $1280 \times 800$. The tablet has an Intel囚 core ${ }^{\mathrm{TM}}$ i7 $\mathrm{CPU}$ with $2.67 \mathrm{GHz}$ and 4GB RAM. The original HP digital stylus that came with the tablet was used for input. Sessions were recorded using screen capture and sound. The task templates on the tablet interface were as big as they would be on a small display device; i.e. none of the templates exceeded $5 \mathrm{~cm}$ in height and width.

\subsection{Distortion Lenses}

We tested three distortion lenses which allowed the participants to sketch in a magnified region. The distortion lenses were implemented into our test environment. The lens size, the shaded regions and the magnification factor were the same for each lens. The lens's width is one third of the canvas height. For interaction support, the lens region is shaded grey and surrounded by blue borders to indicate the lens's shape and size. The magnification factor is three. Besides the distortion technique, different lens shapes are tested.

\subsubsection{Fisheye Lens}

The fisheye lens is based on Carpendale and Montagnese's work (2001). It is divided into the focus and transition regions (Figure 1 (d)). Both regions have the same width. The focus region is flat and shows the ink magnified and undistorted. The transition region uses a linear drop-off function (see below) to distort the ink. The distance between the original point's location $(P)$ and its distorted location $\left(P^{\prime}\right)$ is calculated as follows:

$$
d\left(P, P^{\prime}\right)=\frac{d(C, P)-r_{1} / z}{r_{2}-r_{1} / z} *\left(r_{2}-r_{1}\right)+r_{1}
$$

$C$ is the lens center, $z$ the zoom factor, $r_{1}$ the radius of the focus region, $r_{2}$ the radius of the transition region and $d(C, P)$ the distance between lens center and $P$. The lens shape is rectangular using a $L(\infty)$ metric (Carpendale and Montagnese 2001).

\subsubsection{Blending Lens}

The blending lens is based on Sigma lenses (Pietriga and Appert 2008). It uses different levels of opaqueness to transition between focus and context regions (Figure 3 ). The lens consist of two 
layers: the lower layer shows the original ink while the upper layer shows the magnified ink. The magnified ink is fully opaque within a predefined ( $1 / 3$ of the lens size) distance of the center: outside this area the opacity decreases linearly until it vanishes. The lower layer is designed the opposite way; the ink is fully opaque on the outside of the lens and decreases linearly till it completely vanishes at the lens center. The upper and lower layers use the same linear function to compute each pixel's opacity as the fisheye lens uses to calculate each pixel's offset. The lens shape is rectangular using a $\mathrm{L}(\infty)$ metric.

\subsubsection{Fixed Lens}

The fixed lens is a fisheye lens (Furnas 1986) that can be pinned to a location using the stylus button. Thus, it avoids the known problems of a moving canvas (Gutwin 2002). Pinning the lens decouples stylus and lens movement and allows users to draw freely without the lens moving. When finished, a user unpins the lens using the stylus button and moves it to the next location. The fixed lens's implementation is identical to the fisheye except for the circular shape and ability to be pinned down.

\subsection{Procedure}

Participants sat in front of a desk on which the tablet lay. The tablet screen was folded over the keyboard so that the screen was flat on the table facing the participant. At the start of the study each participant was given a tutorial. After the participants had read the general instructions, they had to complete the first part of the questionnaire.

The next steps were repeated for all three lenses. First, the study facilitator activated the lens and demonstrated its characteristics. Before a lens was used for the first time, the participants familiarized themselves with the lens by playing a game of noughts and crosses against the facilitator. Before and after each evaluation task the facilitator loaded the template and saved the final sketch.

Each participant started with the first task of a task series. Once they completed both drawing tasks, participants rated their experience using the questionnaire. Then the two writing tasks were completed before finishing the rating section of the questionnaire. After completing all 12 sketch tasks, participants used the third questionnaire section to rank the lenses and answered questions regarding preferred and non-preferred lens features.

\subsection{Tasks}

Each participant completed a task series (two drawing and two writing tasks) with each distortion lens (Figure 4). To compel participants to use the lenses, they were given a template for each task, to which they had to add objects. Participants were

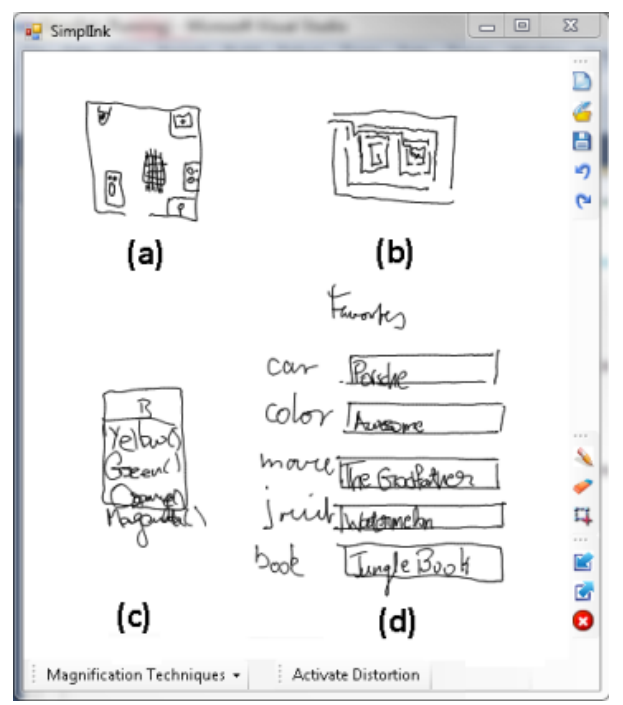

Figure 4: Test environment showing completed examples of the tasks. The upper row shows the drawing tasks ((a) floor plan, (b) maze) and the lower row the writing tasks ((c)UML class diagram, (d) favourites list).

asked not to let added components touch their containing objects. For example, when adding a TV to a TV cabinet, the TV boundaries should not intersect with the cabinet. For the first task in each task series a floor plan had to be completed by adding seven new objects. The challenge for this task was to add small objects to a given scenario.

The second task in each task series asked the participants to complete a maze. The template showed the outer border of the maze with two openings. To complete the tasks further walls had to be progressively added within the inner region forcing the participant to draw increasingly smaller elements. Finally, a line indicating a path from outside the maze to the smallest, innermost shape was drawn. The design of this drawing task forced longer lines to be drawn; the distortion lens was not always big enough to cover the ink of interest completely.

The first writing task contained a skeleton of a UML class diagram to which five method names had to be added. Participants had to ensure each method name did not touch another name or the class box.

The template for the final writing task contained five textboxes to which words had to be added. This task design helped participants with navigation but forced them to move more into writing operations.

\subsection{Data Captured}

For each task we measured the completion time and error rate. Completion time was defined as the period between the first time the stylus was in range until the last object of the task was completely drawn. A shape irregularly intersecting with another shape was counted as an error. A 
Table 1: The distortion lenses including their main features and the results from the evaluation studies.

\begin{tabular}{|l|l|l|l|l|}
\hline \multirow{2}{*}{ Features } & Lens Movement & Comprehension aiding Features & \multicolumn{2}{|c|}{ Evaluation } \\
\cline { 3 - 6 } & & & $\begin{array}{l}\text { Time } \\
\text { (Means) }\end{array}$ & $\begin{array}{l}\text { Errors } \\
\text { (Means) }\end{array}$ \\
\hline Fisheye & Unrestricted & None & 379 & 2.1 \\
\hline Blending & Unrestricted & None & $349 \mathrm{~s}$ & 2 \\
\hline Fixed & $\begin{array}{l}\text { Manually pinned for } \\
\text { sketching }\end{array}$ & None & $463 \mathrm{~s}$ & 0.6 \\
\hline $\begin{array}{l}\text { Auto } \\
\text { zoom }\end{array}$ & $\begin{array}{l}\text { Manually pinned for } \\
\text { sketching }\end{array}$ & Automatically adjust size & $364 \mathrm{~s}$ & 0.2 \\
\hline Semantic & $\begin{array}{l}\text { Only moves on } \\
\text { horizontal axis }\end{array}$ & Highlight semantic groups & $391 \mathrm{~s}$ & 1.4 \\
\hline Snap & $\begin{array}{l}\text { Auto pinned for } \\
\text { sketching }\end{array}$ & $\begin{array}{l}\text { Automatically maximizes shape } \\
\text { coverage }\end{array}$ & $413 \mathrm{~s}$ & 0.3 \\
\hline
\end{tabular}

questionnaire was used to, first, establish the participants' familiarity with digital sketching and distortion lenses, second, to rate different lens characteristics and, third, to capture their likes and dislikes of the lenses. The latter often evolved into a discussion between the facilitator and participant about the effect of the different features.

\subsection{Results}

It took participants approximately one hour to complete the study. We found time taken was normally distributed. Error rates were transformed using the natural logarithm to overcome their nonnormal distribution. The data was analysed using a repeated measures ANOVA for factors distortion lens (fisheye, translucency or fixed lens) and task (drawing or writing). If $p<0.05$ the difference was considered statistically significant. We examined significant differences between groups using Bonferroni-corrected pairwise comparisons. If spericity was violated, Greenhouse-Geisser estimates were used for correction.

The completion time showed a main effect, $F(2,22)=6.338, \quad p<.01$. Pairwise comparisons identified the fixed lens $(M=463 \mathrm{~s})$ as being significantly slower than the blending lens $(\mathrm{M}=349 \mathrm{~s}), \mathrm{p}<.05$ (Table 1). Repositioning required the fixed lens to be pinned and unpinned causing the disadvantageous time difference.

\subsubsection{Questionnaire}

When asked for their preferences, participants ranked the blending lens (50\%) the highest for drawing closely followed by the fixed lens $(41.67 \%)$. For writing the fixed lens was clearly preferred (83.3\%). Participants, on average, agree somewhat to reusing their preferred lens (Figure 5). For willingness to reuse and ease of use, the fixed lens was rated the highest (Figure 5). Yet for both questions, the fixed lens received an average rating of 4.9, just below agree somewhat. One quarter of the participants strongly disagreed to reuse any lens with a moving canvas (fisheye and blending lens). The use of the transition region showed two very different patterns. Overall the rating shows a neutral use of ink in the transition region $(M=4.04)$. However, the high standard deviation (STD=1.87) indicates that the distorted ink was either used a lot or not at all (Figure 5).

The error rate was found to have a main effect, $F(2,22)=63.186, \quad p<.001$. Pairwise comparisons showed that using the fixed lens $(M=0.6)$ resulted in significantly fewer errors than the fisheye $(M=2.1, p<.001)$ and blending lens $(M=2 ; p<.001)$. The higher error rates were caused by the moving canvas during the sketching operations.

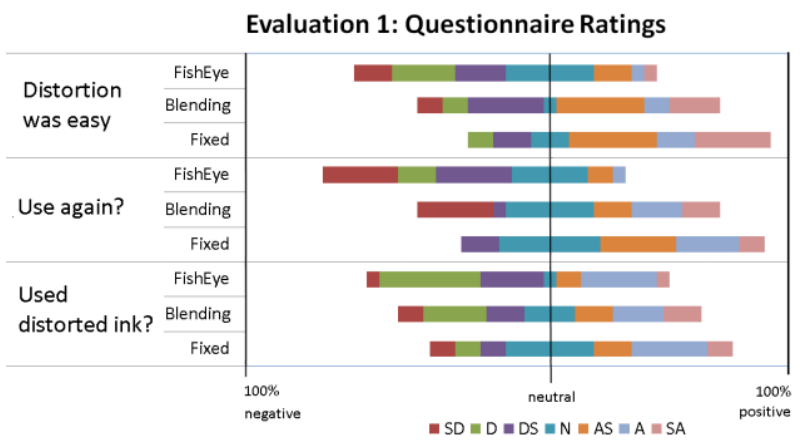

Figure 5: Ratings on a 7 point Likert scale centered on neutral. The scale categories are: strongly disagree (SD), disagree (D), disagree somewhat (DS), neutral ( $N)$, agree somewhat $(A S)$, agree $(A)$ and strongly agree $(S A)$.

\subsubsection{Participants' Comments}

Participants said that the perfect distortion lens should have the ability to be pinned down. Ten participants said pinning down the lens enabled them to draw more space efficiently and neater as the canvas was stationary. They described the feeling of sketching on a moveable canvas as unnatural, undesirable and extremely uncomfortable. The lens preferences and willingness to use a lens again were congruent. Five participants suggested the lens was always a bit too small so they desired an easy way to manipulate the lens size. The different lens shapes were not noticed by ten of the participants. When 
(a)

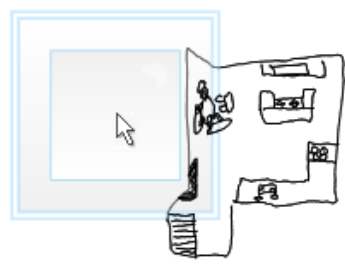

(b)

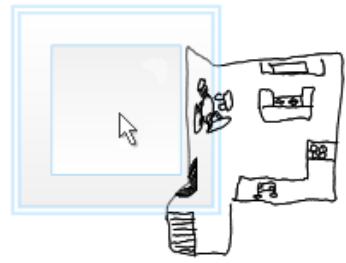

(c)

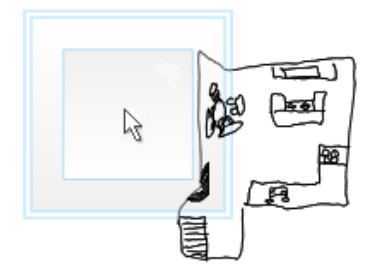

\section{$t_{\mathrm{t} 1}$}
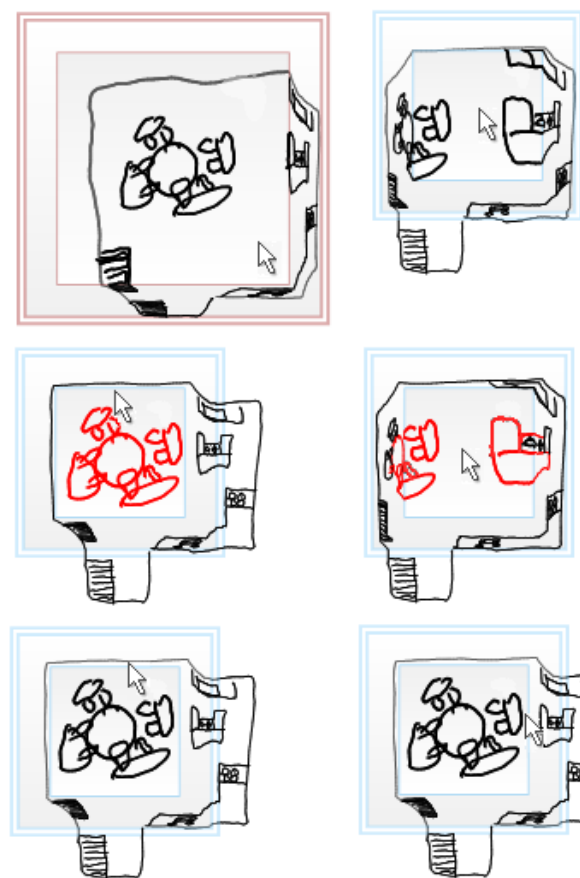
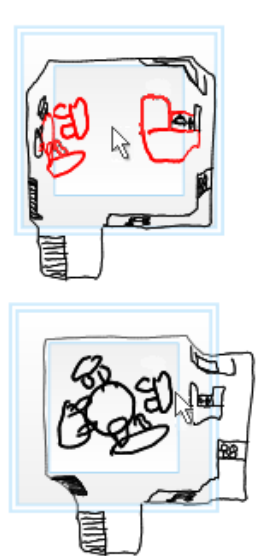
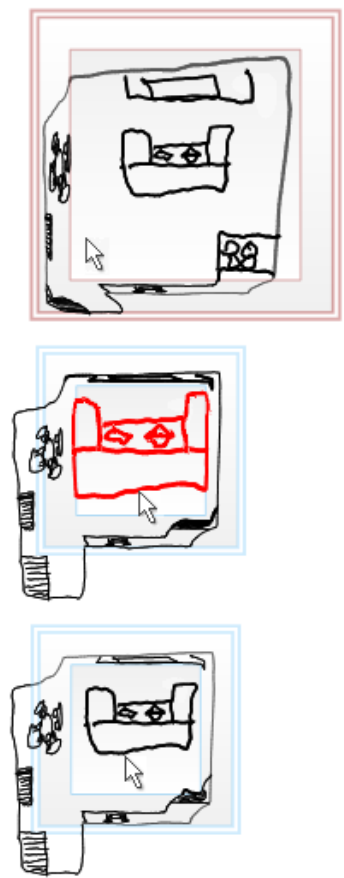

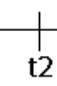

t3

t4 time

Figure 6: Behaviour of the fisheye lens based (a) auto zoom lens, (b) semantic lens and (c) snap lens. The auto zoom lens approaches the sketch ( $t 1)$. When pinned down (t2 \& t4) the lens analyses the covered ink and automatically increases in size to cover all ink of interest. Colouring the lens borders red indicates its pinned state. When the lens is unpinned it assumes its original size and border colour (t3). The semantic lens moves continuously horizontally (t1-t4). When drawing (t2 \& t4) the cursor moves freely vertically without the lens following. The semantic lens highlights the strokes belonging to the same group (t2). As the lens is navigated to the right (t3) the group changes (t4). As the snap lens approaches the sketch (t1) it snaps onto the first group and the cursor can be moved freely (t2). As the cursor continues moving to the right the lens remains on its initial position (t3) until the cursor leaves the inner lens and it snaps onto the next group in reach

specifically asked, nine said they did not care and three stated a preference for the rectangular shape. The fixed lens was preferred by $83.3 \%$ of the participants for writing. When asked why, eleven out of twelve participants specifically stated that the stationary canvas was their main reason. However a fixed lens made drawing larger shapes more cumbersome as they frequently had to reposition the lens. The missing ability of the fixed lens to move freely led to the lower ranking for drawing. The blending and fisheye lens offered a free moving lens at the cost of a moving canvas. However, a typical comment when using a lens with a moving canvas was: "it feels like writing in a moving bus." Participants also stated that the fisheye's distorted ink and the blending lens's unmagnified ink were difficult to comprehend.

In sum, comments such as "the winning lens wasn't really good by itself but was the best one of the three." suggested further research needed to be done before distortion lenses could be used to successfully support magnified sketching. Although none of the existing lenses proved suitable, using them provided insight into the usefulness of their individual features. We believe that the fisheye technique has the most potential as it better visually integrates the magnified ink into the nonmagnified sketch. (t4).

\section{INTELLIGENT NEW LENSES}

Building on the results from the first evaluation study, we implemented three novel distortion lenses (Figure 6). Each of the new lenses explores different solutions for two of the problems identified in the first study: first, the moving canvas when sketching and, second, to make the distorted ink more appealing to use.

\subsection{Distortion Lenses}

To solve the problem of the moving canvas, three different strategies to optimize stylus and lens coupling were implemented. Also, to minimize the impact of distortion, the new lenses use context information from the sketch to regulate what is distorted and emphasize shapes of importance. The three different coupling and context highlighting strategies are combined in different ways in each lens. The new lenses are based on the fisheye lens from the first evaluation: if not stated otherwise, they are identical in size and use the same linear drop off function. Table 1 summarizes the approaches.

\subsubsection{Auto Zoom Lens}

The auto zoom lens can be manually pinned to a spot thus avoiding the moving canvas problem. Pinning a lens decouples stylus and pen enabling 
the pen to move without the lens following. It remains at the location until it is unpinned. The pinned state is visualized by the magnifier's border being red instead of blue (Figure $6(\mathrm{a})$ ). To address the context issue when the auto zoom lens is pinned, ink in the magnified area is analysed. If the lens covers most of an underlying shape(s) the lens's size is automatically adjusted to cover it completely. Intelligently increasing the lens size reduces the likelihood of ink of interest being in the transition region. Thus the required difficult mental mapping between a shape's distorted and magnified view are alleviated.

A busy canvas may lead to difficulties for the algorithm to find a clear size boundary. To solve this, the lens can grow at most up to one quarter of the window size. This may lead to shapes of interest being in the transition region but means distorted ink isn't made unrecognizable.

\subsubsection{Semantic Lens}

The moving canvas problem is addressed by partially decoupling the lens and stylus when sketching: the semantic lens only follows the stylus on the horizontal axis when sketching. As the stylus vertical position changes, the lens's vertical position remains the same so no canvas movement on the vertical axis occurs. If the stylus is above the canvas the lens moves freely in all directions.

To support context understanding this lens highlights ink belonging to a group (Figure 6 (b)). To identify related strokes, semantic rules and recognition algorithms are used. The semantic rules are based on stroke type and spatial layout. There are two types of strokes: drawing and writing. A recognition algorithm from Patel et al. (Patel, Plimmer et al. 2007) is used to determine a stroke's type. This was chosen based on accuracy and classification time. The algorithm was originally used to classify strokes after they were completely drawn. To adapt the algorithm to recognize unfinished strokes, we undertook an empirical study. The aim was to determine the minimum number of stoke points required by the recognition algorithms. We found that after seven points the recognition algorithm correctly classified the strokes in $88 \%$ of all cases. Note that we only considered cases when the complete stroke was correctly classified.

The stroke types together with the distances between strokes were used to determine whether a currently drawn stroke belongs to an existing semantic group. If a stroke is within a calculated distance from the previously drawn stroke it belongs to the same semantic group as the previous one. The distance is based on the average length of the strokes already in the semantic group. If the distance is smaller than $1 / 5$ of the average length then the currently sketched stroke is automatically in the group. If it is smaller than $1 / 3$ of the average length and the previously stroke is of type writing, the stroke is also part of the semantic group. The latter is necessary as text consists of smaller strokes than shape strokes. If the currently sketched stroke does not belong to an existing group it forms its own group.

\subsubsection{Snap Lens}

To overcome the moving canvas problem, the snap lens analyses the underlying ink to automatically pin itself to a location (Figure 6 (c)). Gravity fields are used to decide when and where the lens snaps. The snapping algorithm calculates an invisible gravity field for each stroke based on the stroke's size and its spatial environment. Whenever the lens enters a gravity field, it automatically positions itself in the field's center. The size of the gravity field is determined by a shape's size and the free space around it.

There are two snapping strategies depending on the targeted shape's spatial environment. If it is empty (i.e. if a lens snapped over a shape doesn't cover any other shape), the shape's gravity field's size is the sum of its bounding box and the lens's radius. If there are other shapes in a shape's environment, their gravity fields are merged. If a gravity field is too big to be covered completely by a lens, it is divided into smaller, non-overlapping, rectangular fields. Each field has the size of the magnified lens region. The merged gravity field is divided in a left to right, top to bottom manner.

To release the snap, the pen has to be moved outside the magnified region. However, a snap is only released if the user is not sketching. This strategy of snap and release favours fewer but more distant lens repositionings over more frequent, smaller ones. In an initial study this behaviour was preferred by participants as more frequent repositionings interrupted thought and sketch processes. When no existing ink is within a predefined lens radius, the lens is moved freely. If the lens snaps to an undesired location, the pen button can be used to overrule the snap. To support context understanding, the snap lens assumes an optimized position over the shapes. The aim is to maximize shape coverage and minimize the ink in the transition region, similar to the auto zoom lens.

\section{EVALUATION}

To be able to compare the new lenses with those of the previous study, we evaluated them using the same methods, procedure, measured variables and tasks. The new lenses have the same initial size (one third of the canvas height), magnification factor (3) and shaded regions as the lenses in the 
first study. Additionally, using fisheye distortion for all lenses (as it seems to have more potential) makes it easier to isolate other features' effects.

\subsection{Participants}

Twelve participants volunteered to complete the four tasks using the three new lenses. None of the participants for this study took part in the previous study. Their age range was between 22 and 34 years. Two participants were female and three were left-handed.

\subsection{Results}

The completion times showed no main effect when looking at the new lenses, $F(2,22)=1.927, p=.169$. There were also no main effects when comparing the lenses from the first and second study, $F(5,55)=2.519, p=.086$, and between the lenses with a stationary canvas $F(3,33)=2.524, p=.120$; i.e. fixed $(M=463 \mathrm{~s})$, auto zoom $(M=364 \mathrm{~s})$, snap $(M=413)$ and semantic lens $(M=391)$. Neither can a main effect be found when comparing the fastest lens with (auto zoom lens) and without (blending lens) a stationary canvas, $F(1,11)=0.121, p=.735$, even when looking at task 1 which involves a lot of repositionings, $F(1,11)=.499, p=.494$. Overall, the new lenses don't require significantly more time despite the decoupling of lens and stylus.

The error rate showed a main effect for the new lenses, $\quad F(2,22)=30.914, \quad p<.0001$. Pairwise comparisons showed that using the auto zoom $(\mathrm{M}=0.2, \mathrm{p}<.0001)$ and snap lens $(\mathrm{M}=0.3, \mathrm{p}<.0001)$ results in significantly fewer errors than the semantic lens $(M=1.4)$.

There was also a main effect when comparing the six lenses from both studies, $F(5,55)=31.960$, $\mathrm{p}<.0001$. Pairwise comparisons showed that the lenses offering a completely stationary canvas produced significantly fewer errors than those which do not; i.e. fixed $(M=0.6)$, auto zoom $(M=0.2)$ and snap lens $(M=0.3)$ allow for fewer errors than fisheye $(M=2)$, blending $(M=2)$ and semantic lens $(M=1.4)$. We conclude that it is not sufficient to restrict the canvas movement as the semantic lens resulted in significantly more errors than those with a stationary canvas, $\mathrm{F}(3,33)=9.712, \mathrm{p}<.003$.

For both drawing and writing the auto zoom lens was preferred by $75 \%$ of participants followed by the snap lens. The semantic lens was never rated as the preferred lens. The main reason participants gave for disliking it was the moving canvas, not the semantic highlighting.

When asked which features to include into a perfect lens seven participants chose the snap function over the pin one which received only two votes, the remaining three did not comment on this.

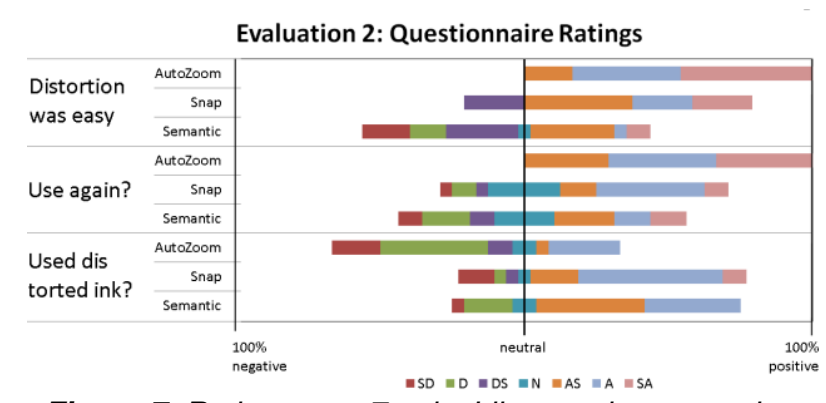

Figure 7: Ratings on a 7 point Likert scale centered on neutral. The scale categories are as in Figure 5.

The auto zoom function was liked by eight participants.

Participants were more likely to use the distorted ink when using either snap or semantic lens (Figure 7 ). With the auto zoom we observed that while drawing participants tended to reposition the lens whenever something of interest was not in the magnified region thus avoiding using the distorted ink.

\section{DISCUSSION}

Current distortion techniques are unsuitable for sketching. Participants rated their willingness to use any of them again as neutral at most (Figure 5 ). Besides the unsuitability of existing distortion techniques there are three key findings from our evaluations: new design guidelines; the success of intelligent distortion lenses; and the similarities between drawing and writing.

\subsection{New Design Guidelines}

There are four features of lenses on which we can comment: canvas movement, transition region, lens shape and distortion technique. The evaluation studies highlight that it is critical to overcome the moving canvas problem and to choose efficient mechanisms to determine what to magnify and how to emphasize shapes of interest.

A moving canvas while sketching caused significantly more errors than a stationary canvas. A main effect was found for the error rate across all six lenses from both evaluations. Even limiting the canvas movement to one direction as is done with the semantic lens resulted in a significantly higher error rate. One approach to overcome the moving canvas problem was to decouple the lens and stylus movement. When done manually (fixed lens) more time was required to complete the tasks. A snapping mechanism to automatically decouple the lens and stylus has no negative impact on the task completion time. Our snapping lens was received positively: the majority of participants voted the snap feature into their perfect lens. 
Transition regions are an undoubted benefit of distortion lenses (Gutwin and Skopik 2003; Cockburn, Karlson et al. 2009). However they do increase cognitive load. This is particularly a problem when the item of interest spans the magnified and transition regions. The ability to intelligently increase the magnification region size is one approach to tackle this problem. By covering the ink of interest in the magnified region, users do not need to spend time to comprehend the distorted ink in the transition region. This is evident when comparing the completion times of auto zoom lens $(M=98 \mathrm{sec})$ and the fixed lens $(M=143 \mathrm{sec})$ for writing tasks.

The other approach to the transition region issue we trialled was to highlight shapes of interest in the semantic lens. The idea was to highlight these groups to further support content understanding when sketching. Coping with the movement of the lens overwhelmed the other features of the semantic lens. When asked whether the highlighted ink helped them in understanding the context, most participants said that they did not pay much attention to the groups. One participant commented that colouring the groups was perceived as insufficiently different from the other ink that "it did not really stand out".

We are not confident that this highlighting had a fair trial as it was a part of a moving lens. A more aggressive solution could be to only magnify ink of interest. This could be done using the blending lens distortion techniques which are based on translucency. The semantic group would be shown on the upper layer magnified while the rest is shown with increasing levels of translucency at normal size in the background.

Our first evaluation suggested that people found fisheye more natural and comprehendible than blending. With fisheye there are a range of different functions that can be used in the transition region (Carpendale and Montagnese 2001). Our only exploration into this was in an early prototype where we used an exponential function. Informal studies showed that people had difficulty with translating the distances as consistent with (Gutwin 2002). Currently we use a linear function, but this is an area worthy of further exploration.

\subsection{Success of Intelligent Distortion Lenses}

The new lenses were perceived as more satisfying than existing ones. Comparing the first two questions in Figure 5 and Figure 7 it is evident the new lenses were easier to use, more likely to be reused and their distorted ink was perceived as more helpful. In the first study each lens roughly received equally negative and positive ratings (Figure 5). The new lenses (Figure 7) were much more positively scored. The auto zoom lens had all positive scores, while the snap lens was mainly positive. The semantic lens, with its partly moving canvas was rated the worst of the new lenses reinforcing the problem of a moving canvas.

\subsection{Similarities between Writing and Drawing}

The last key finding is that we could not find large differences in requirements for drawing and writing. An evaluation study by Lank and Phan (2004) suggests drawing and writing are two different activities so may require different lenses. In our study we asked participants to rate the lenses for drawing and writing individually. Despite participants choosing different lenses as their favourites for drawing and writing, we have not found great differences between the questionnaire ratings for the activities.

\section{CONCLUSION \& FUTURE WORK}

Much research has been done into magnification techniques in general and distortion lenses specifically. Distortion lenses have been proven successful for a variety of tasks (Baudisch, Good et al. 2002; Gutwin and Skopik 2003; Cockburn, Karlson et al. 2009). However, the contexts to which distortion lenses have been applied are content navigation and selection, not creation and editing. We applied distortion lenses to sketching using them to create and edit content in form of digital ink. We undertook two stages of evaluation: first, evaluating three current distortion lenses for sketching to investigate performance and the general requirements of this domain on distortion lenses. Second, using the results of the first study we designed and evaluated three intelligent distortion lenses.

The results show that current distortion lenses are unsuitable for sketching. Two major problems current techniques face are the moving canvas and the decision of what to magnify and how. The new lenses demonstrate that distortion lenses that overcome such limitations can successfully support magnified sketching. The intelligent lenses' novel techniques solve the moving canvas problem via pinning. Automatic pinning has a higher user acceptance as it relieves the tedium of manual pinning. This and the lenses' use of context information resulted in higher user acceptance and ease of use. The new lenses also resulted in a lower error rate without impacting completion times.

Our findings extend existing research in that we apply distortion lenses to a new domain, thereby exposing them to new challenges. Considering the individual success of the features, we can say with confidence that the lens should have a snapping function so that there is a stationary canvas, an auto resize function and to optimize the ink coverage. The solutions found in this research can 
be applied to solve problems found with distortion lenses in existing research.

\section{REFERENCES}

Agrawala, M. and M. Shilman. (2005) DIZI: A Digital Ink Zooming Interface for Document Annotation INTERACT, Rome, Italy, 12-16 Sep, 6979. Springer, Heidelberg, Germany.

Appert, C., O. Chapuis and E. Pietriga. (2010) High-precision magnification lenses. Proceedings of the 28th international conference on Human factors in computing systems, Atlanta, USA, 10-15 Apr, 273-282. ACM, New York, USA.

Baudisch, P., N. Good, V. Bellotti and P. Schraedley. (2002) Keeping things in context: a comparative evaluation of focus plus context screens, overviews, and zooming. Proceedings of the SIGCHI conference on Human factors in computing systems, Minneapolis, Minnesota, USA, 20-25 Apr, 259-266. ACM, New York, USA.

Bederson, B. B. (2000) Fisheye menus. Proceedings of the 13th annual ACM symposium on User interface software and technology, San Diego, California, United States, 5-8 Nov, 217-225. ACM, New York, USA.

Carpendale, M. S. T., D. J. Cowperthwaite and F. D. Fracchia. (1997) Making distortions comprehensible. Visual Languages, 1997. Proceedings. 1997 IEEE Symposium on, Capri, Italy, 23 -27 Sep, 36-45. IEEE Computer Society, Washington, USA

Carpendale, M. S. T. and C. Montagnese. (2001) A framework for unifying presentation space. Proceedings of the 14th annual ACM symposium on User interface software and technology, Orlando, Florida, 11-14 Nov, 61-70. ACM, New York, USA.

Cockburn, A., A. Karlson and B. B. Bederson. (2009) A review of overview+detail, zooming, and focus+context interfaces. ACM Comput. Surv., 41, 1-31.

Furnas, G. (1986) Generalized fisheye views. Proceedings of the SIGCHI conference on Human factors in computing systems, Boston, USA, 13-17 Apr, 16-23. ACM, New York, USA.

Gatward, K., (2008) Effects of canvas size on sketching: Implications for Creativity Support Tools, The University of Bath, Bath, UK.

Grudin, J. (2001) Partitioning digital worlds: focal and peripheral awareness in multiple monitor use. Proceedings of the SIGCHI conference on Human factors in computing systems, Seattle, Washington, United States, 1-5 Apr, 458-465. ACM, New York, USA.

Gutwin, C. (2002) Improving focus targeting in interactive fisheye views. Proceedings of the SIGCHI conference on Human factors in computing systems: Changing our world, changing ourselves, Minneapolis, Minnesota, USA, 20-25 Apr, 267-274. ACM, New York, USA.
Gutwin, C. and A. Skopik. (2003) Fisheyes are good for large steering tasks. Proceedings of the SIGCHI conference on Human factors in computing systems, Ft. Lauderdale, Florida, USA, 5-10 Apr, 201-208. ACM, New York, USA.

Hornbaek, K. and E. Frokjaer. (2003) Reading patterns and usability in visualizations of electronic documents. ACM Trans. Comput.-Hum. Interact., 10, 119-149.

Kosara, R., S. Miksch and H. Hauser. (2001) Semantic Depth of Field. Proceedings of the IEEE Symposium on Information Visualization 2001 (INFOVIS'01), San Diego, USA, 21-26 Oct, 97. IEEE Computer Society, Washington, USA.

Lank, E. and S. Phan. (2004) Focus+Context sketching on a pocket PC. $\mathrm{CHI} \mathrm{C}^{\circ} 4$ extended abstracts on Human factors in computing systems, Vienna, Austria, 24-29 Apr, 1275-1278. ACM, New York, USA.

Patel, R., B. Plimmer, J. Grundy and R. Ihaka. (2007) Ink Features for Diagram Recognition. 4th Eurographics Workshop on Sketch-Based Interfaces and Modeling Riverside, California, 2-3 Aug, 131-138. ACM, New York, USA.

Pietriga, E. and C. Appert. (2008) Sigma lenses: focus-context transitions combining space, time and translucence. Proceeding of the twenty-sixth annual SIGCHI conference on Human factors in computing systems, Florence, Italy, 5-10 Apr, 1343-1352. ACM, New York, USA.

Plimmer, B. and I. Freeman. (2007) A Toolkit Approach to Sketched Diagram Recognition. $\mathrm{HCl}$, Lancaster, UK, 3-7 Sep, 205-213. eWiC, London, UK.

Plimmer, B., H. C. Purchase and H. Y. Yang. (2010) SketchNode: Intelligent sketching support and formal diagramming. OZCHI Brisbane, Australia, 26-30 Nov, 136-143. ACM, New York, USA.

Ramos, G., A. Cockburn, R. Balakrishnan and M. Beaudouin-Lafon. (2007) Pointing lenses: facilitating stylus input through visual-and motorspace magnification. Proceedings of the SIGCHI conference on Human factors in computing systems, San Jose, California, USA, 28 Apr-3 May, 757-766. ACM, New York, USA.

Robertson, G. G. and J. D. Mackinlay. (1993) The document lens. Proceedings of the 6th annual ACM symposium on User interface software and technology, Atlanta, Georgia, United States, 3-5 Nov, 101-108. ACM, New York, USA.

Schaffer, D., Z. Zuo, S. Greenberg, L. Bartram, J. Dill, S. Dubs and M. Roseman. (1996) Navigating hierarchically clustered networks through fisheye and full-zoom methods. ACM Trans. Comput.-Hum. Interact., 3, 162-188.

Wickens, C. D. and J. G. Hollands. (2000). Decision Making. Engineering Psychology and Human Performance. Prentice Hall, Upper Saddle River, NJ, USA. 